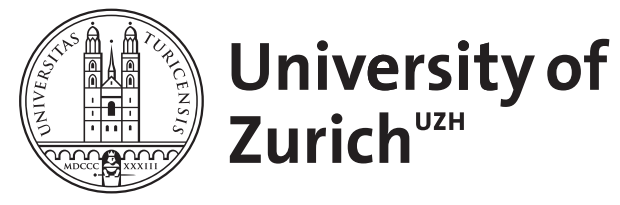

\title{
Nicht invasive Überwachung der Herzfunktion mit Thoracocardiographie
}

\author{
Bloch, K E ; Russi, E W
}

DOI: https://doi.org/10.1515/bmte.1996.41.s1.528

Posted at the Zurich Open Repository and Archive, University of Zurich ZORA URL: https://doi.org/10.5167/uzh-154610

Journal Article

Published Version

Originally published at:

Bloch, K E; Russi, E W (1996). Nicht invasive Überwachung der Herzfunktion mit Thoracocardiographie. Biomedizinische Technik. Biomedical engineering, 41(s1):528-529.

DOI: https://doi.org/10.1515/bmte.1996.41.s1.528 


\title{
Nicht invasive Überwachung der Herzfunktion mit Thoracocardiographie
}

\author{
Bloch K.E., Russi E.W. \\ Abteilung für Pneumologie, Universitätsspital Zürich, CH-8091 Zürich, Schweiz
}

\section{EINLEITUNG:}

Thoracocardiographie ist eine neue, nicht invasive Methode zur Überwachung der mechanischen Herzfunktion[1-3]. Ein induktionsplethysmographischer Sensor, der auf Höhe des Herzens um den Thorax gelegt wird, registriert Volumen-Änderungen, welche durch die Atmung und Herztätigkeit entstehen. Kardiale werden von respiratorischen Signalanteilen durch digitale Filterung und EKG-getriggerte Mittelung getrennt. Damit können segmentale Volumenänderungen des Herzens (VentrikelVolumenkurven) aufgezeichnet werden. Ihre Amplitude entspricht dem Herzschlagvolumen. Durch zusätzliche Messung der Herzfrequenz kann das Herzminutenvolumen (HMV) kontinuierlich überwacht werden. Ziel dieser Studie war eine Überprüfung der Präzision der Thoracocardiographie zur HMV-Messung im Vergleich zur Thermodilution.

\section{METHODE:}

Bei 21 schwerkranken Patienten auf der Intensivstation wurden simultane Messungen des HMV mit Thoracocardiographie und Thermodilution durchgefuihrt. Da Thoracocardiographie lediglich relative Änderungen, nicht aber absolute Werte des HMV (in L/Min.) registriert, wurde das erste Messwertepaar pro Patient zur Eichung der Thoracocardiographie verwendet und von der weiteren Auswertung ausgeschlossen. Alle darauffolgenden HMV-Werte wurden in L/Min. ausgedrückt.

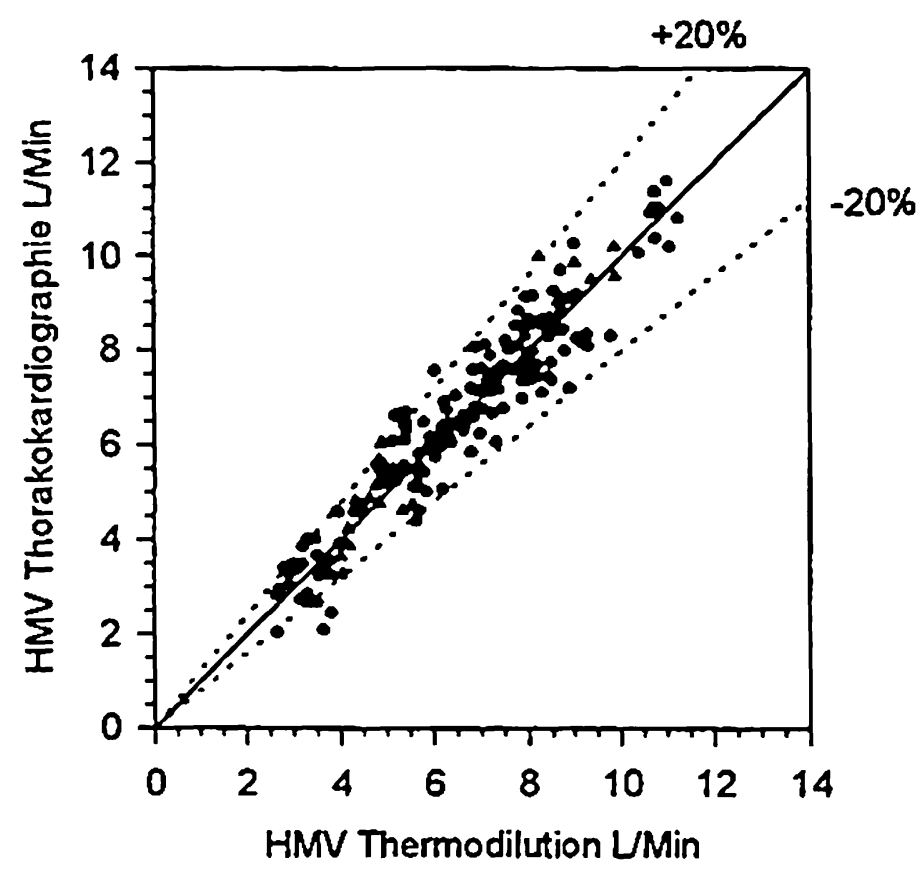

Figur 1: Vergleich der mit den beiden Methoden gemessenen Herzminutenvolumina

\section{ERGEBNISSE:}

Bei 201 simultanen Messungen mit Thoracocardiographie und Thermodilution standen nach Ausschluss der ersten Messung pro Patient noch 180 Messwertepaare zum Vergleich zwischen den Methoden zur Verfugung. Die Beobachtungsdauer pro Patient erstreckte sich über 35 bis 254 Min. Der Bereich der HMV-Werte (Thermodilution) betrug 2.7 bis $11.3 \mathrm{~L} / \mathrm{Min}$. Die mittlere Differenz (bias) des HMV(Thoracocardiographie-Thermodilution) betrug 0.0 L/Min. Der $95 \%$ Vertrauensbereich der mittleren Differenz (limits of agreement) betrug $\pm 0.8 \mathrm{~L} / \mathrm{Min}$. oder $\pm 21 \%$. Die mittlere Differenz der mit beiden Methoden gemessenen HMV-Änderungen betrug $0 \%$ (95\% Vertrauensbereich -21 bis $+22 \%$ ) bei HMV-Änderungen von -34 bis $+56 \%$.

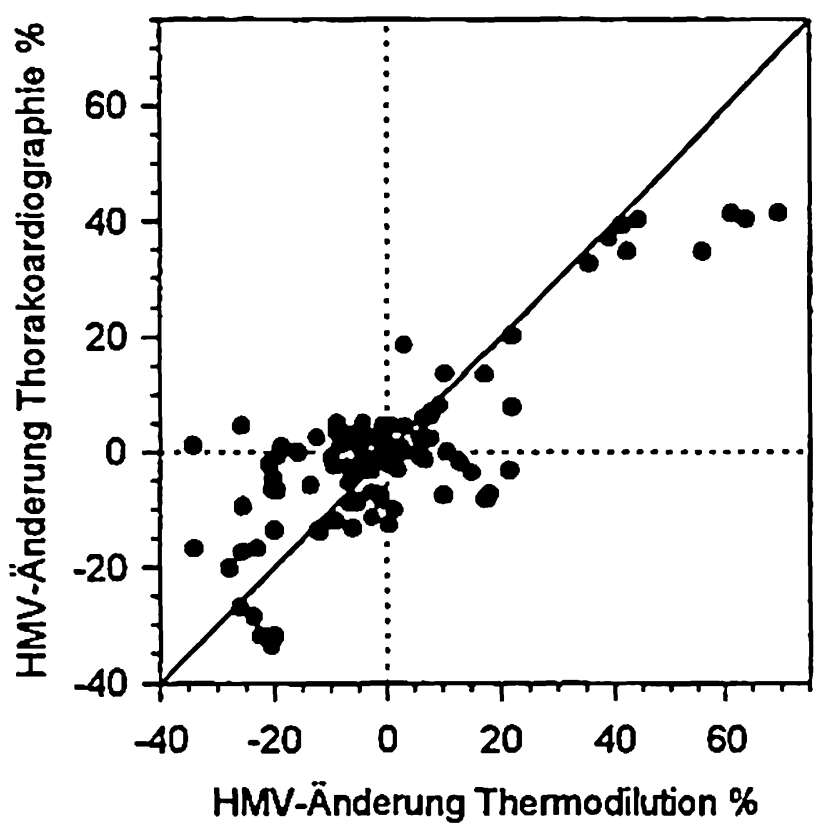

Figur 1: Vergleich der mit den beiden Methoden gemessenen Änderungen des Herzminutenvolumens

\section{DISKUSSION:}

Die Abweichung der thoracocardiographischen von den mittels Thermodilution gemessenen HMV-Werten war von ähnlicher Grössenordnung wie diejenige zwischen Thermodilution und anderen HMV-Messmethoden. Die Präzision der Thoracocardiographie zur Messung des HMV ist für klinische Anwendungen brauchbar. Die Methode ermöglicht eine nicht invasive Überwachung der mechanischen Herzfunktion bei schwerkranken Patienten unter Bedingungen einer Intensivstation.

\section{LITERATUR:}

[1] M.A. Sackner et al.; Chest 99, 613 (1991)

[2] K.E. Bloch et al.; Chest 106, 1668 (1994)

[3] K.E. Bloch et al.; Am J Respir Crit Care Med; in press (1996) 
